\title{
Progress of Interference of Traditional Chinese Medicine on Cirrhosis Treated with Bone Marrow Mesenchymal Stem Cells
}

\author{
Yaxin Wang $\mathbb{D},{ }^{1}$ Huicun Zhang $\mathbb{D},{ }^{1,2}$ and Hongbing Wang $\mathbb{D}^{1,3}$ \\ ${ }^{1}$ Beijing Hospital of Traditional Chinese Medicine, Capital Medical University, Beijing 100010, China \\ ${ }^{2}$ Beijing Institute of Traditional Chinese Medicine, Beijing 100010, China \\ ${ }^{3}$ Beijing Hospital of Traditional Chinese Medicine Yanqing Hospital, Beijing 102199, China \\ Correspondence should be addressed to Huicun Zhang; zhanghuicun728@126.com and Hongbing Wang; ivy0106@sina.com
}

Received 12 February 2021; Revised 18 April 2021; Accepted 4 May 2021; Published 11 May 2021

Academic Editor: Harry Lee

Copyright ( $) 2021$ Yaxin Wang et al. This is an open access article distributed under the Creative Commons Attribution License, which permits unrestricted use, distribution, and reproduction in any medium, provided the original work is properly cited.

Transplantation of bone marrow mesenchymal stem cells has attracted more and more attention as a regenerative therapy for the treatment of liver diseases. A large number of studies have shown that this kind of cells can inhibit the activation of hepatic stellate cells and regulate tissue homeostasis and immune system via a variety of ways. Meanwhile, bone marrow mesenchymal stem cells can inhibit apoptosis of hepatocyte, improve liver function, and reduce inflammation through multiple pathways. These cells have a broad prospect in the treatment of liver cirrhosis. At present, there are many studies on the specific mechanism of bone marrow mesenchymal stem cells transplantation in the treatment of liver cirrhosis. This paper reviews the pathogenesis of liver cirrhosis and the mechanism of bone marrow mesenchymal stem cells transplantation in the treatment of liver cirrhosis, discusses the effectiveness of traditional Chinese medicine method in enhancing the efficacy of bone marrow mesenchymal stem cells transplantation, and looks forward to its application prospect in the future.

\section{Introduction}

Liver cirrhosis is the end stage of chronic liver disease. The progress of chronic liver disease can cause a variety of body damage, such as upper gastrointestinal bleeding, infection, hepatic encephalopathy, hepatocellular carcinoma, hepatorenal syndrome, and other malignant diseases [1]. Liver cirrhosis, contributing to more than 1 million deaths annually, is a major healthcare concern worldwide [2]. Patients with end-stage liver cirrhosis often require liver transplantation. But this treatment is faced with many problems that are difficult to solve, for example, the shortage of liver source, high immune rejection, being prone to postoperative complications [3], and high cost associated with the operation, so its clinical application is limited [4]. In order to prevent liver disease from developing into its end-stage, mesenchymal stem cell (MSC) therapy has been adopted and has shown its potential in curing this kind of liver disease. As an individualized cell therapy, MSCs transplantation has been deeply studied and has a broad prospect of clinical application. Bone marrow mesenchymal stem cells (BMSCs), of which the genetic background is relatively stable and the use rarely involves ethical problems, can be easily isolated and greatly expanded in vitro. By using these cells, the implantation reaction in vivo is weak, the rejection of transplantation can be avoided, and its allogeneic cell transplantation does not pose a risk of xenovirus infection. It has attracted much attention on how to improve the survival rate, increase proliferation ability of transplanted BMSCs, induce it to differentiate into mature hepatocytes, change the damaged liver microenvironment, and make it safe for clinical use. This paper reviews the mechanism of BMSCs transplantation in the treatment of liver cirrhosis and looks forward to its application prospect in the treatment of liver disease.

1.1. MSCs: Biology and Origins. In 1976, Friedenstein et al. [5] first expanded stromal cells obtained from marrow cell suspensions in vitro and identified discrete colonies of plastic-adherent. Each colony was named a colony-forming 
unit fibroblast (CFU-F). Cells that produced CFU-F colonies were named osteogenic stem cells or bone marrow stromal stem cells [6]. These cells exhibited a huge in vitro expansion and a potential to differentiate into adipocytes, chondrocytes, and osteocytes. The isolation of MSCs according to current criteria produces heterogeneous, non-clonal cultures of stromal cells containing stem cells with different multipotential properties, committed progenitors, and differentiated cells. To improve the characterization of MSCs, the International Society for Cellular Therapy (ISCT) proposed three rules to identify MSCs. According to the ISCT, MSCs must be purified from the bone marrow stromal population based on plastic adherence under standard culture conditions; MSCs must express the CD105, CD90, and CD73 antigens. Moreover, they should be CD45 (-) and CD34 (-). In addition, MSCs must differentiate in vitro into osteocytes, chondrocytes, and adipocytes [7]. The characteristics of MSCs above make them play an important role in clinic and experiment. Now most of the MSCs used in the study of liver cirrhosis are isolated from bone marrow. Using BMSCs in the treatment of liver cirrhosis has the following advantages: (1) they have a wide range of sources and are convenient to obtain; (2) they have a high ability of selfrenewal and can be expanded massively in vitro; (3) they can differentiate into a variety of tissue types under the induction of appropriate culture medium and factors; (4) the multidirectional differentiation characteristics of MSCs will not be affected after being transferred into exogenous genes; (5) there are no problems such as tissue matching and immune rejection. The study of the mechanism of BMSCs in the treatment of liver cirrhosis is helpful to solve the existing problems.

1.2. Pathogenesis of Liver Cirrhosis. Liver cirrhosis is the final pathological result of various chronic liver diseases, and fibrosis is the precursor of cirrhosis. According to the existing research, the central link of liver cirrhosis is the activation of hepatic stellate cells (HSCs). When multiple molecular signal pathways in HSCs are activated, the molecular biological characteristics of hepatic stellate cells can be changed at the gene level, which can induce liver fibrosis. HSCs reside in the space of Disse in the normal liver and their main function is storage of Vitamin A and other retinoids [8]. Normally, HSCs are in a state of rest; when the liver is injured by inflammation or mechanical stimulation, pro-inflammatory factors such as platelet-derived growth factor (PDGF), tumour necrosis factor- (TNF-) $\alpha$, transforming growth factor- (TGF-) $\beta$, and interleukin- (IL-) 1 can activate HSCs. After HSCs are activated, they will lose the oil droplets storing Vitamin A, proliferate under the stimulation of growth factors, and differentiate into myofibroblasts. Activated HSCs move to the injured site induced by monocyte chemoattractant protein-1 (MCP-1) and chemokine ligand 2 (CXCR2) and synthesize a large amount of extracellular matrix (ECM) and fibrotic collagen (I, II). The activity of matrix metalloproteinases (MMPs) will be inhibited and the degradation of ECM will be reduced by the overexpression of tissue matrix metalloproteinase inhibitors
(TIMPs) [9]. The synthesis of ECM, which is mainly composed of collagen, was increased, while the degradation was relatively insufficient, and the total collagen could be increased to 3-10 times of the normal. Too much collagen deposited in the Disse space, resulting in the widening of the gap and the formation of the basement membrane under the hepatic sinusoid endothelial cells (LSECs). The reduction or even disappearance of the superior window pore of the endothelial cells would form a diffuse barrier and a capillarization of the hepatic sinusoid [10]. With the flattening of villi on the surface of hepatocytes and the formation of barriers, the transport of substances in hepatic sinusoids through the walls of hepatic sinusoids to hepatocytes was blocked, which interfered with the function of hepatocytes directly, resulting in the dysfunction of synthesis of hepatocytes. The stenosis of hepatic sinusoid, obstruction of blood flow, and increase of intrahepatic resistance affect the hemodynamic of portal vein, cause hypoxia of hepatocytes and disturbance of nutrient supply, aggravate hepatocyte necrosis, and make initiator factors work continuously. The fibre bundle of the portal area and hepatic capsule extend to the central vein of the hepatic lobule. These fibre septa encircle the regenerated nodules or re-segment the residual hepatic lobules and transform them into pseudo-lobules, forming a typical histopathological morphology of liver cirrhosis.

\section{Mechanism of BMSCs in Liver Cirrhosis}

Transplantation of BMSCs into damaged liver to reconstruct liver structure and improve liver function is of great significance for the treatment of liver fibrosis and liver cirrhosis. The mechanism of BMSCs in the treatment of liver cirrhosis is reviewed below. It is pointed out that there are still some outstanding problems of BMSCs transplantation in the treatment of liver cirrhosis.

2.1. Inhibiting the Activation of Hepatic Stellate Cells. The activation of HSCs is an important factor in hepatic fibrosis. The main function is to synthesize ECM components in normal liver tissue or liver cirrhosis. We know that liver cirrhosis is the repair response of the liver to various acute and chronic stimulation injuries. In the process of liver injury, HSC is an important cell for hepatocytes to produce ECM. BMSCs can inhibit the proliferation and activation of HSCs. Sakaida [11] confirmed that portal vein injection of BMSCs can inhibit the activity of HSCs and the expression of inflammatory molecules, protect the function of mitochondria, reduce oxidative stress, and thus improve the regeneration of rat hepatocytes and reduce the fibrosis of regenerated liver. Previous studies have shown that growth factors and cytokines secreted by BMSCs can inactivate HSCs and inhibit the progress of liver fibrosis. In in vitro experiments, the co-culture of BMSCs and HSCs can lead to cell cycle arrest in G10/G1 phase and cell apoptosis and ultimately inhibit the proliferation of HSCs [12]. Cao et al. [13] found that BMSCs can inhibit the expression of actin alpha 2 (ACTA2) and hepatocyte growth factor (HGF), 
initiate paracrine action, secrete a variety of anti-fibrosis cytokines and growth factors, induce HSCs apoptosis, reduce the deposition of ECM, and inhibit the formation of liver inflammation and fibrosis.

2.2. Participating in Immune Regulation. Liver fibrosis is the inevitable result of liver damage and chronic inflammation, and it is the necessary stage for the development of liver cirrhosis. Any cause of liver inflammation is characterized by inflammatory cell infiltration. The immunoregulatory properties of BMSCs are facilitated by their interactions with immune cells like $\mathrm{T}$ cells, B cells, dendritic cells, macrophages, and natural killer (NK) cells in a context and microenvironment-dependent manner [14]. The pro-fibrotic factors secreted by these cells play an important role in the activation and proliferation of HSCs [15]. These cells interact with liver parenchymal cells or nonparenchymal cells and jointly participate in the formation and regulation of liver inflammation and fibrosis. BMSCs secrete various soluble mediators, inhibit the proliferation and activation of various immune cells, and ultimately inhibit the activation of hepatic stellate cells. Research by Guo et al. found that MSCs transplantation in patients with liver cirrhosis can inhibit the proliferation of peripheral blood $\mathrm{T}$ lymphocytes, upregulate the expression of CD4, CD25, and CD127, regulatory T cells (Treg), and also alleviate the Treg/Th17 imbalance [16]. Liu et al. [17] also found that, during liver fibrosis, CD4+, $\mathrm{CD} 25+$, FoxD3, and other regulatory $\mathrm{T}$ cells in the liver increased, while NK cells decreased. CD4+, CD25+, and Foxp3+ cells are recognized regulatory $\mathrm{T}$ cell subsets in recent years, and they are the maintainers of peripheral immune tolerance. They can also inhibit or release IL-10, TGF- $\beta$, and other cytokines through cell-to-cell contact to inhibit the effect of $\mathrm{T}$ cells, which prevents the body from getting out of control. Xin et al. [18] found that BMSCs can inhibit the activation of $\mathrm{T}$ lymphocytes and inhibit the immune response by reducing the release of the inflammatory factor TNF- $\beta$. In addition, BMSCs can downregulate immune response and produce immune tolerance through autocrine and inducing $\mathrm{T}$ regulatory cells to produce and secrete immunosuppressive factors IL-10 and TGF- $\beta$. Macrophages play a central role in liver fibrosis and fibrosis regression [19]. In the process of liver fibre formation, proinflammatory M1 macrophages located near activated liver myofibroblasts secrete pro-fibrotic factors. This secretion promotes the increase of myofibroblast fibrogenesis, activation, proliferation, and chemotaxis. However, studies [20] have shown that MSCs can induce changes in cytokineactivated macrophages and promote fibrosis resolution.

2.3. Promoting the Degradation of Collagen Fibres. There are kinds of MMPs in the liver, of which the main function is to degrade extracellular matrix. The activation of MMPs is related to TIMPs. Under normal circumstances, metalloproteinases in hepatocyte matrix can degrade collagen fibres in hepatocyte matrix. When liver cirrhosis occurs, the TIMP secreted by activated HSCs increases and inhibits the activity of MMP, leading to a large increase of collagen fibres in liver tissue [21]. Khalifa et al. [22] used superparamagnetic nanometre iron oxide (SPIO) to label BMSCs and found that BMSCs can promote the regression of fibrous tissue in liver fibrosis. MMP-1 can degrade type I and III collagen, which is the key collagen in irreversible scars of liver cirrhosis. Matrix metalloproteinase-9 (MMP-9) specifically degrades type III collagen and gelatin to control the progression of liver fibrosis [23]. Du et al. [24] have studied the therapeutic effect of BMSCs transfected with human matrix metalloproteinase-1 (MMP-1) on carbon tetrachloride-induced hepatic fibrosis in rats. They suggested that BMSCs may be a potential cell source in preventing liver fibrosis and MMP1 gene may enhance the anti-fibrotic effect of BMSCs. The in vitro studies of Tanimoto et al. [25] found that BMSCs inhibit the activation of HSCs by regulating the expression of humoral factors and increase MMP-9, which helps to inhibit activated HSCs and improve fibrosis.

2.4. Beneficial to Hepatocyte Regeneration. Related studies have found that, under specific culture conditions, BMSCs can change their phenotype and play a metabolic function similar to that of hepatocytes. Some studies have shown that administered BMSCs can migrate to the liver [26]. However, direct transplantation of MSCs is available, but the number of hepatocytes in vivo that differentiate from MSCs transplanted is very low. Liver cells derived from MSCs are less than $1 \%$ of total liver cells, so liver function regeneration is extremely limited [27], which is still a clinical obstacle to BMSCs transplantation. BMSCs after transplantation have low survival rate and short half-life in host tissues. More recently, a number of other findings have indicated that the beneficial effects of MSC transplants should be ascribed to the capacity of MSCs to regulate tissue homeostasis and the immune system via a plethora of cytokines, growth factors, and differentiation factors [6]. At present, some studies have found that more BMSCs can be transformed into hepatocytes by some methods. Li et al. [28] found that autologous BMSCs can differentiate into hepatocytes and promote the regeneration of residual liver after portal vein embolization in patients with liver cirrhosis. In vitro, BMSCs can be induced to differentiate into precocious hepatocyte-like cells (HLCs) in indirect co-culture system with primary hepatocytes [29]. The mechanism may be to improve the microenvironment by alleviating liver cirrhosis and upregulating the expression of vascular endothelial growth factor, hepatocyte growth factor, IL-10, and MMP-9. The regeneration of hepatocytes is closely related to the repair of liver injury. Hu et al. [30] found that overexpression of liver nuclear factor- (HNF-) $4 \alpha$ directly induced immortalized human BMSCs to form HLCs. Li et al. [31] found that ultrasound radiation can induce BMSCs to transform into hepatocytes. Tan et al. found that Galectin-3 can induce rat BMSCs to differentiate into HLCs, and the differentiation efficiency can be improved further when combining with HGF. Inhibition of HIPPO signal pathway can improve the induction efficiency [32]. These studies provide a new strategy for the clinical application of BMSCs transplantation in the future. 
2.5. Effect of Traditional Chinese Medicine on BMSCs in the Treatment of Liver Cirrhosis. Traditional Chinese medicine has a potential targeting function because of its unique views on disease, meridian tropism of traditional Chinese medicine, and promoting the operation of qi, blood, and body fluid, especially in the treatment of liver cirrhosis by tonifying kidney and activating blood circulation and removing blood stasis. At present, there are more and more clinical studies on traditional Chinese medicine combined with stem cell transplantation, and there are more and more cases of traditional Chinese medicine to improve the success rate of transplantation and liver function. The mechanism and the best dose will be increasingly clear. Traditional Chinese medicine can directly repair liver injury from multiple angles or indirectly improve the recovery of liver function by promoting BMSCs function or regulating transplanted cell colonization and immune system, so traditional Chinese medicine combined with stem cell transplantation will have a broader development prospect in the treatment of liver diseases.

2.6. Promoting the Proliferation of BMSCs. The therapeutic effect of BMSCs transplantation is closely related to the number of successful survival cells after transplantation. A large number of animal experiments and cytological studies have found that traditional Chinese medicine and its drugcontaining serum can promote the proliferation of stem cells and increase the number of surviving cells after cell transplantation, thus improving the therapeutic effect. Wang et al. [33] studied the effects of different concentrations of Ligustrazine on the proliferation and adhesion of BMSCs and found that Ligustrazine can promote the proliferation of BMSCs and that appropriate concentration of Ligustrazine can significantly improve the adhesion of BMSCs. Ligustrazine is an alkaloid isolated from the rhizome of chuanxiong, which provides a reliable basis for clinical application of chuanxiong to regulate the proliferation of BMSCs and improve the adhesion of BMSCs. Chen et al. [34] found that the effective concentration range is $0.01 \sim 3.0 \mathrm{mg} /$ $\mathrm{mL}$ tortoise plate extract, which can increase the proportion of BMSCs in the proliferative phase and promote the proliferation of BMSCs. Huang et al. [35] studied the effect of different concentrations of astragalus polysaccharides on the proliferation of BMSCs and found that $1-3 \mathrm{mg} / \mathrm{mL}$ astragalus polysaccharides could promote the proliferation of BMSCs, in which the upregulation of SCF mRNA and SCF protein content may be the mechanism of astragalus polysaccharides promoting the proliferation of BMSCs in vitro. Peng et al. [36] found that astragaloside can promote the proliferation of human BMSCs in vitro, and its mechanism may be related to its promotion for the expression of SCF, VEGF, and SDF-1 mRNA in human BMSCs, which provides a theoretical basis for astragalus membranaceus to play a role in BMSCs transplantation. Icaritin (ICT) is an active ingredient of the genus Epimedium, a traditional Chinese medicine, with the potential to enhance the proliferation of MSCs. Lu et al. [37]found out that MSCs increased the survival rate of acute liver failure rats and reduced liver damage. MSCs cocultured with ICT exerted a greater therapeutic effect than MSCs alone. Further, the $\mathrm{HGF} / \mathrm{c}-$ Met pathway played a key role in the antiapoptotic activity of MSCs, which was associated with the optimized efficacy of ICT.

\section{Improving BMSCs Differentiating into HLCs}

Chinese Materia Medical can not only stimulate the proliferation of BMSCs and improve the survival rate of BMSCs, but also improve the success rate of MSCs differentiating into HLCs. The differentiation of MSCs has been seen in two main routes: (1) directly into hepatocytes; (2) induced by cytokines such as hepatocyte growth factor (HGF) that is secreted by MSCs and injured liver tissue. Curcumin is the main active ingredient extracted from turmeric, which has the function of promoting blood circulation, removing blood stasis, promoting qi, promoting menstruation, relieving pain, and so on, so it is widely used in the treatment of liver diseases. Chen et al. [38] found that $20 \mathrm{ng} / \mathrm{mL}$ curcumin can induce BMSCs to differentiate into HLCs. To make it safer and better, we can combine with FGF-4 so as to reduce the concentration of curcumin. Cordyceps polysaccharide is the main effective component of Cordyceps sinensis. Liu et al. [39] found that, 14 days after induction, alpha-fetoprotein, albumin, keratin 18, and glycogen staining were all positive in Cordyceps polysaccharide group. It is confirmed that Cordyceps polysaccharides can induce rat BMSCs to differentiate into HLCs in vitro. Liu et al. [39] studied the effects of Cordyceps polysaccharides combined with BMSCs transplantation on the expression of MMP-13 and TIMP-1 in cirrhotic rats and found that Cordyceps polysaccharides combined with MSCs transplantation could reduce the expression of TIMP-1 in liver tissue, further enhance the expression of MMP-13, significantly promote collagen degradation, and reduce collagen deposition. Ye and Wang [40] found that the combined treatment of rhubarb and MSCs can promote hepatocyte regeneration and repair after liver injury.

3.1. The Mechanism of Herbal Formulas in the Treatment of Liver Fibrosis. Yiguanjian decoction is a famous prescription for the treatment of liver disease, which comes from the "Continued Cases of Well-Known Physicians" (a collection of medical records in ancient China). It was founded by Yupu Wei, a famous doctor in the Qing Dynasty, and is composed of Coastal Glehnia Root, Radix Ophiopogonis, Angelica sinensis, Radix Rehmanniae, Chinese wolfberry, Toosendan, and other herbs. It has been proved to reduce liver fibrosis. Fu et al. [41] found that Yiguanjian decoctions drug-containing serum could facilitate the differentiation of murine BMSCs into hepatocytes in vitro and has a synergistic effect with SDF-1 and HGF. By exploring the related mechanism of inducing BMSCs to differentiate into hepatocytes, Ping et al. [42] found that Yiguanjian decoctions serum can increase the expression of Alb, AFP, and HNF4a mRNAs of BMSC and promote the expression of CK18 protein. It is proved that Yiguanjian decoctions can induce 
BMSCs to differentiate into hepatocytes which has the function of mature hepatocytes. The mechanism may be related to the downregulation of $\mathrm{Wnt} / \beta$-catenin signal pathway. Because of the therapeutic effect of this decoction on liver cirrhosis, the study of its mechanism in the treatment of liver cirrhosis is helpful to better apply it in the treatment of liver diseases. Chen et al. [43] found that fuzhenghuoxue formula enhanced hepatocyte differentiation from human embryonic stem cells. These enhancements by fuzhenghuoxue formulas are mediated through activation of canonical Wnt pathways and ERK pathways and inhibition of Notch pathway. Li et al. [44] studied the effect of zuoguiwan drug-containing serum on the differentiation into hepatocytes. They believe that kidney-tonifying prescriptions can promote the transformation of BMSCs into hepatocytes, regulate systemic function, and improve liver microenvironment. Wen et al. [45] explored the mechanism of biejiajianwan intervention on BMSCs through Notch signal pathway. It was found that biejiajianwan can promote the differentiation of BMSCs into HLCs, and its mechanism may be related to the regulation of Notch-1 protein and Notch-1mRNA expression in BMSCs Notch signal pathway. Wen et al. [45] believe that biejiajianwan combined with bone marrow stem cell transplantation can improve the effect of treatment of liver fibrosis, and it may work through the action of SDF-1/CXCR4 axis.

\subsection{Problems and Prospect of BMSCs Transplantation in the} Treatment of Liver Cirrhosis. Autologous BMSCs transplantation may be the best choice for cell therapy in patients with liver cirrhosis [46]. However, the uncertainty is how to make BMSCs play a successful and effective role in clinical application. Scholars have made relevant clinical studies on the role of BMSCs in the treatment of liver cirrhosis. It is still difficult to distinguish how many hepatocytes in patients are regenerated by BMSCs [29]. In spite of this, there are still many reports about the successful clinical application of BMSCs. There are several noteworthy areas in clinical trials that need to be further addressed, such as the best cell type, the best timing of treatment, the most effective cell count, and the best number of injections [47]. Besides, the survival time of transplanted cells is also important and we need to achieve a sustained effect. This requires immunohistochemical analysis of human-specific markers to observe these cells [48]. However, for clinical use, more complex techniques will need to be developed. For example, transplanted cells can be labelled so that they can be tracked. So, it is necessary to have advanced imaging techniques. These require constant exploration. In addition to the clinical efficacy of BMSCs in the treatment of liver cirrhosis, there are other problems that need to be solved urgently. Some studies have pointed out that MSCs may promote tumour growth in vivo, so people question the long-term effectiveness and potential carcinogenic risk of MSCs therapy. On the other hand, some studies have suggested that MSCs may have anti-tumour effect, which is related to the regulation of inflammatory environment of many tumours. MSCs can also interact with cancer cells and inhibit signal pathways related to tumour growth and cell division, so the safety of BMSCs transplantation also needs to be monitored. At the same time, the isolation, culture, and identification of BMSCs in vitro need to be further improved, so that they can better differentiate and expand into hepatocytes. About the indications and contraindications of direct transplantation and transplantation after vitro differentiation of BMSCs, and how to avoid vascular embolism in cell transplantation, these problems need to be further observed, solved, and improved in the treatment of liver cirrhosis with BMSCs. At present, how to use BMSCs safely and effectively in the treatment of end-stage liver disease is a key research field of many scholars. For various ways of using BMSCs in the treatment of liver cirrhosis, the research of traditional Chinese medicine provides some new clues. As we all know, traditional Chinese herbs monomer and traditional Chinese medicine prescription have the characteristics of "multi-component, multi-target, multi-channel, multi effect." Therefore, to explore the target of traditional Chinese medicine intervention on BMSCs in the treatment of liver cirrhosis is helpful to focus on effective prevention and treatment of traditional Chinese medicine or prescription and provide scientific theoretical basis for clinical treatment of liver fibrosis and reversal of liver cirrhosis. However, the composition of traditional Chinese medicine is complex, and its specific mechanism of action and target still need to be further explored. Some traditional Chinese medicine prescriptions even contain hepatotoxic drugs. How to control the dosage in the process of use and how to reduce its toxic and side effects are also issues that need further study.

\section{Conclusions}

BMSC therapy is a promising cell therapy, which can treat liver fibrosis and reverse liver cirrhosis through a variety of ways, and has a certain therapeutic effect. In the subsequent experiment, how to modify BMSCs with safety and stability, as well as how to induce those cells to play a greater role in the treatment of liver cirrhosis, is a problem that still needs to be solved. Traditional Chinese medicine has its unique advantages in controlling the progression of liver cirrhosis and relieving clinical symptoms. The research on the intervention of MSCs in the treatment of liver cirrhosis by traditional Chinese medicine has received great attention and achieved many achievements. It is necessary to systematically sort out the theoretical basis of traditional Chinese medicine in the intervention of liver cirrhosis and MSCs to better expand the research ideas. We should also deeply excavate the effective components of traditional Chinese medicine, properly use the achievements of modern science and technology, and increase the research on the MSCs regulated by traditional Chinese medicine, in order to improve the ability of traditional Chinese medicine in liver diseases. It is believed that, with the further deepening of relevant research, traditional Chinese medicine will play a key role in the research and application of stem cells, which is very attractive for the development of effective anti-fibrosis therapy. Therefore, the study of BMSCs in the treatment of liver cirrhosis needs to be more in-depth and do 
a good job in basic research, so as to contribute to improving the therapeutic effect and quality of life of patients with liver disease.

\section{Data Availability}

The data used to support the findings of this study are available from the corresponding author upon request.

\section{Conflicts of Interest}

The authors declare that there are no conflicts of interest regarding the publication of this paper.

\section{Acknowledgments}

This work was supported by the National Nature Science Foundation of China (grant no. 81873244) and the Fund for Beijing Science \& Technology Development of TCM (grant no. JJ2018-30).

\section{References}

[1] K. Nishikawa, Y. Osawa, and K. Kimura, "Wnt/ $\beta$-Catenin signaling as a potential target for the treatment of liver cirrhosis using antifibrotic drugs," International Journal of Molecular Sciences, vol. 19, no. 10, p. 3103, 2018.

[2] M. Rybicka, A. Woziwodzka, A. Sznarkowska et al., "Liver cirrhosis in chronic hepatitis b patients is associated with genetic variations in DNA repair pathway genes," Cancers, vol. 12, no. 11, p. 3295, 2020.

[3] M. Linecker, T. Krones, T. Berg et al., "Potentially inappropriate liver transplantation in the era of the "sickest first" policy - a search for the upper limits," Journal of Hepatology, vol. 68, no. 4, pp. 798-813, 2018.

[4] S. Zhang, Y. Yang, L. Fan, F. Zhang, and L. Li, "The clinical application of mesenchymal stem cells in liver disease: the current situation and potential future," Annals of Translational Medicine, vol. 8, no. 8, p. 565, 2020.

[5] A. J. Friedenstein, J. F. Gorskaja, and N. N. Kulagina, "Fibroblast precursors in normal and irradiated mouse hematopoietic organs," Experimental Hematology, vol. 4, no. 5, pp. 267-274, 1976.

[6] U. Galderisi and A. Giordano, "The gap between the physiological and therapeutic roles of mesenchymal stem cells," Medicinal Research Reviews, vol. 34, no. 5, pp. 1100-1126, 2014.

[7] M. Dominici, K. Le Blanc, I. Mueller et al., "Minimal criteria for defining multipotent mesenchymal stromal cells. The International Society for Cellular Therapy position statement," Cytotherapy, vol. 8, no. 4, pp. 315-317, 2006.

[8] W.-C. Zhou, Q. B. Zhang, and L. Qiao, "Pathogenesis of liver cirrhosis," World Journal of Gastroenterology, vol. 20, no. 23, pp. 7312-7324, 2014.

[9] S. Huang and X. Xue, "Pathogenesis and treatment progress of liver fibrosis," Guangdong Chemical Industry, vol. 46, no. 9, pp. 145-146+107, 2019.

[10] K. Böttcher and M. Pinzani, "Pathophysiology of liver fibrosis and the methodological barriers to the development of antifibrogenic agents," Advanced Drug Delivery Reviews, vol. 121, pp. 3-8, 2017.
[11] I. Sakaida, "Autologous bone marrow cell infusion therapy for liver cirrhosis," Journal of Gastroenterology and Hepatology, vol. 23, no. 9, pp. 1349-1353, 2008.

[12] L. Wang, G. Bai, and F. Chen, "Human bone marrow mesenchymal stem cells suppress the proliferation of hepatic stellate cells by inhibiting the ubiquitination of p27," Biochemistry and Cell Biology, vol. 95, no. 6, pp. 628-633, 2017.

[13] H. J. Cao, M. D. Wang, S. G. Li, L. Zhu, and J. H. Zheng, "Paracrine effect of bone marrow mesenchymal stem cells on proliferation, apoptosis, and alpha-actin-2 expression in hepatic stellate cells," Genetic Molecular Research, vol. 16, no. 1, 2017.

[14] A. Mohanty, N. Polisetti, and G. J. J. o. b. Vemuganti, "Immunomodulatory properties of bone marrow mesenchymal stem cells," Journal of Biosciences, vol. 45, 2020.

[15] G. Kim, K. Y. Shim, and S. K. Baik, "Diagnostic accuracy of hepatic vein arrival time performed with contrast-enhanced ultrasonography for cirrhosis: a systematic review and metaanalysis," Gut and Liver, vol. 11, no. 1, pp. 93-101, 2017.

[16] C. H. Guo, L. X. Han, M. R. Wan, G. J. Deng, and J. H. Gan, "Immunomodulatory effect of bone marrow mesenchymal stem cells on $\mathrm{T}$ lymphocytes in patients with decompensated liver cirrhosis," Genetics and Molecular Research, vol. 14, no. 2, pp. 7039-7046, 2015.

[17] X. Liu, J. Lou, Y. Chen, and Z. Duan, "[Changes of regulatory T cells related to $\mathrm{CCl}_{4}$-induced liver fibrosis in mice]," Zhonghua gan Zang Bing Za Zhi, vol. 22, no. 4, pp. 277-280, 2014.

[18] Y. Xin, J. Gao, R. Hu et al., "Changes of immune parameters of T lymphocytes and macrophages in EAE mice after BM-MSCs transplantation," Immunology Letters, vol. 225, pp. 66-73, 2020.

[19] G. Kim, J. H. Huh, K. J. Lee, M. Y. Kim, K. Y. Shim, and S. K. Baik, "Relative adrenal insufficiency in patients with cirrhosis: a systematic review and meta-analysis," Digestive Diseases and Sciences, vol. 62, no. 4, pp. 1067-1079, 2017.

[20] S. H. Kang, M. Y. Kim, and S. K. Baik, "Novelties in the pathophysiology and management of portal hypertension: new treatments on the horizon," Hepatology International, vol. 12, no. S1, pp. 112-121, 2018.

[21] E. Roeb, "Matrix metalloproteinases and liver fibrosis (translational aspects)," Matrix Biology, vol. 68-69, pp. 463473, 2018

[22] Y. H. Khalifa, G. M. Mourad, W. M. Stephanos, S. A. Omar, and R. A. Mehanna, "Bone marrow-derived mesenchymal stem cell potential regression of dysplasia associating experimental liver fibrosis in albino rats," BioMed Research International, vol. 2019, pp. 1-15, Article ID 5376165, 2019.

[23] Y. Iimuro, T. Nishio, T. Morimoto et al., "Delivery of matrix metalloproteinase-1 attenuates established liver fibrosis in the rat," Gastroenterology, vol. 124, no. 2, pp. 445-458, 2003.

[24] C. Du, M. Jiang, X. Wei et al., "Transplantation of human matrix metalloproteinase-1 gene-modified bone marrowderived mesenchymal stem cell attenuates CCL4-induced liver fibrosis in rats," International Journal of Molecular Medicine, vol. 41, no. 6, pp. 3175-3184, 2018.

[25] H. Tanimoto, S. Terai, T. Taro et al., "Improvement of liver fibrosis by infusion of cultured cells derived from human bone marrow," Cell and Tissue Research, vol. 354, no. 3, pp. 717-728, 2013.

[26] L. Li, S. Wu, Z. Liu et al., "Ultrasound-targeted microbubble destruction improves the migration and homing of mesenchymal stem cells after myocardial infarction by upregulating SDF-1/CXCR4: a pilot study," Stem Cells, vol. 2015, Article ID 691310, 14 pages, 2015. 
[27] N. Siedlecka, K. Mecinska-Jundzill, E. Fierek et al., "Biostimulative effect of laser on growth of mesenchymal stem/ stromal cells in vitro," Advances in Dermatology and Allergology, vol. 37, no. 5, pp. 771-780, 2020.

[28] L. Wei, H.-W. Yi, G.-H. Lei, and P. Y. Sheng, "Bone formation in rabbit's leg muscle after autologous transplantation of bone marrow-derived mesenchymal stem cells expressing human bone morphogenic protein-2," Indian Journal of Orthopaedics, vol. 48, no. 4, pp. 347-353, 2014.

[29] I. Ullah, K. Seo, H. Wi, Y. Kim, S. Lee, and S. A. Ock, "Induction of the differentiation of porcine bone marrow mesenchymal stem cells into premature hepatocyte-like cells in an indirect coculture system with primary hepatocytes," Animal Cells and Systems, vol. 24, no. 5, pp. 289-298, 2020.

[30] X. Hu, P. Xie, W. Li, Z. Li, and H. Shan, "Direct induction of hepatocyte-like cells from immortalized human bone marrow mesenchymal stem cells by overexpression of HNF $4 \alpha$," Biochemical and Biophysical Research Communications, vol. 478, no. 2, pp. 791-797, 2016.

[31] F. Li, Y. Liu, Y. Cai et al., "Ultrasound irradiation combined with hepatocyte growth factor accelerate the hepatic differentiation of human bone marrow mesenchymal stem cells," Ultrasound in Medicine \& Biology, vol. 44, no. 5, pp. 10441052, 2018.

[32] M. Tan, Y. Liang, W. Huang et al., "Galectin-3 induces differentiation of rat bone marrow mesenchymal stem cells into hepatocyte-like cells," Nan Fang Yi Ke Da Xue Xue Bao, vol. 38, no. 9, pp. 1076-1082, 2018.

[33] J. Wang, T. Qu, L. Li et al., "Effect of Ligustrazine on proliferation and adhesion of bone marrow mesenchymal stem cells (in Chinese)," Zhejiang Journal of Traditional Chinese Medicine, vol. 51, no. 04, pp. 280-282, 2016.

[34] D. F. Chen, H. P. Zeng, S. H. Du et al., "Extracts from Plastrum testudinis promote proliferation of rat bone-marrow-derived mesenchymal stem cells," Cell Proliferation, vol. 40, no. 2, pp. 196-212, 2010.

[35] J. Huang, J. Zhang, and $\mathrm{Z}$. Xu, "Effect of astragalus polysaccharide on the proliferation of bone marrow mesenchymal stem cells and the expression of stem cell factor in vitro," Fudan University Journal of Medical Sciences, vol. 38, no. 4, pp. 343-348, 2011.

[36] X. Peng, H. Bai, C. Wang, R. Xi, and E. Chao, “"Effect of astragaloside IV on the proliferation and cell factor expression in hBMSC," Progress in Modern Biomedicine, vol. 14, no. 8, pp. 1452-1455, 2014.

[37] L. Wang, S. Li, H.-Y. Wang et al., "In a rat model of acute liver failure, icaritin improved the therapeutic effect of mesenchymal stem cells by activation of the hepatocyte growth factor/c-met pathway," Evidence-Based Complementary and Alternative Medicine, vol. 2019, no. 2, pp. 1-13, 2019.

[38] Y. Chen, W. Zou, S. Wang, and H. Qiao, "Curcumin induces the differentiation of bone marrow mesenchymal stem cells into hepatocytes," Chinese Traditional Patent Medicine, vol. 36, no. 6, pp. 1124-1128, 2014.

[39] J. Liu, Y. Song, Y. Liu, Y. Yang, and J. Zhang, "Cordyceps polysaccharide induces differentiation of adult rat mesenchymal stem cells into a hepatocyte lineage in vitro," Journal of Clinical Rehabilitative Tissue Engineering Research, vol. 49, pp. 221-225, 2012.

[40] D. Ye and F. Wang, "Analysis on the therapeutic effect of rhubarb extract in the model of rats with acute liver failure," China Modern Doctor, vol. 55, no. 12, pp. 32-34, 2017.

[41] L. Fu, B. Pang, Y. Zhu et al., "Ex vivo stromal cell-derived factor 1-mediated differentiation of mouse bone marrow mesenchymal stem cells into hepatocytes is enhanced by Chinese medicine yiguanjian drug-containing serum," Evidence-based Complementary and Alternative Medicine, vol. 2016, Article ID 7380439, 12 pages, 2016.

[42] J. Ping, H. Y. Chen, Y. Zhou, Y. Cheng, and L. M. Xu, "Effect of Yiguan decoction on differentiation of bone marrow mesenchymal stem cells into hepatocyte-like cells: an experimental research," Chinese Journal of Integrated Traditional and Western Medicine, vol. 34, no. 3, pp. 348-354, 2014.

[43] J. Chen, W. Gao, P. Zhou et al., "Enhancement of hepatocyte differentiation from human embryonic stem cells by Chinese medicine Fuzhenghuayu," Scientific Reports, vol. 6, p. 18841, 2016.

[44] H. Li, X. Gao, X. Yan, A. Ming, and Y. Peng, "Study on molecular mechanism of Zuoguiwan in promoting bone marrow cells to form hepatic cells," Journal of Traditional Chinese Medicine, vol. 47, no. 10, pp. 778-780, 2006.

[45] Z. Wen, Z. Wang, J. Huang, M. Xiong, and D. Wei, "Effect of Notch signaling system on directional differentiation of bone marrow mesenchymal stem cells and intervention of Biejiajianwan (in Chinese)," Lishizhen Medicine and Materia Medica Research, vol. 028, no. 12, pp. 2847-2849, 2017.

[46] F. A. M. Abo-Aziza, A. K. A. Zaki, and A. M. Abo El-Maaty, "Bone marrow-derived mesenchymal stem cell (BM-MSC): a tool of cell therapy in hydatid experimentally infected rats," Cell Regeneration, vol. 8, no. 2, pp. 58-71, 2019.

[47] M. Kasraeian, E. Ghasemi, M. Dianatpour et al., "In utero xenotransplantation of mice bone marrow-derived stromal/ stem cells into fetal rat liver: a preliminary study," International Journal of Reproductive BioMedicine, vol. 18, no. 9, 2020.

[48] J. S. Lunn, S. A. Sakowski, and E. L. Feldman, "Concise review: stem cell therapies for amyotrophic lateral sclerosis: recent advances and prospects for the future," Stem Cells, vol. 32, no. 5, pp. 1099-1109, 2014. 